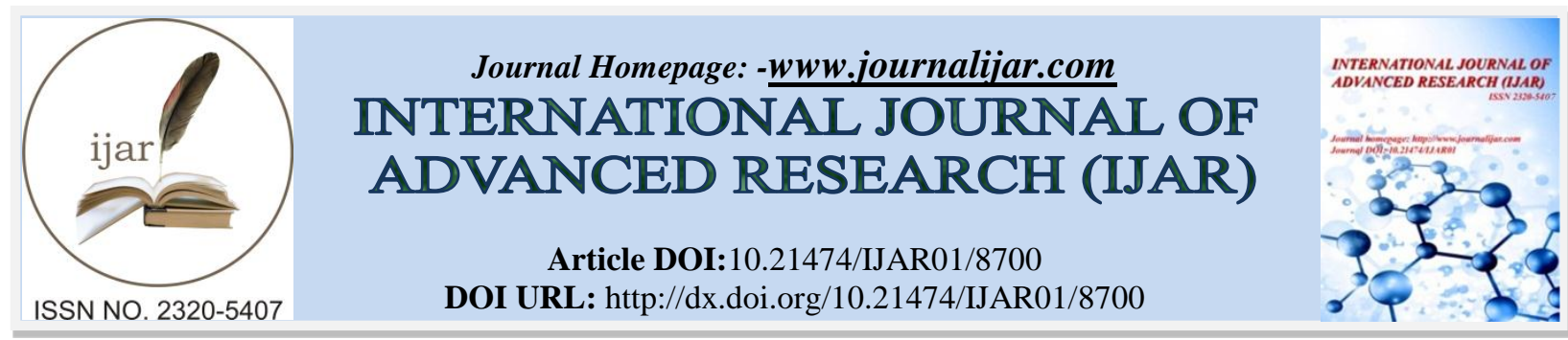

RESEARCH ARTICLE

\title{
THE TREATMENT OF TRAUMATIC BRAIN INJURY IN EXPERIMENTAL ANIMALS BY PYRIMIDINE DERIVATIVES.
}

\section{Voronkov A. V' ${ }^{1}$, M. M. Khaled ${ }^{2}$, Khoury E. $I^{1}$ and Pozdnyakov D. $I^{1}$.}

1. Medical and Pharmaceutical Institute - A branch of state educational institution of higher professional education "Volgograd State Medical University".

2. Medical \& clinical Biochemistry. LU, Faculty of Public Health.

\section{Manuscript Info}

\section{Manuscript History}

Received: 15 January 2019

Final Accepted: 17 February 2019

Published: March 2019

Key words:-

Traumatic Brain Injury (TBI), Cerebral

Edema, Biomarkers, Pyrimidine

Derivatives.

\section{Abstract}

Abstract: Traumatic Brain Injury (TBI) is a significant civilian health problem. TBI triggers complex changes on the central nervous system. NSE and GFAP may provide drug target identification. We aim in this study to investigate the possible effects of pyrimidine derivatives in the recovery of TBI.

Materials and methods: Research was conducted on 80 male animal rats, with body weights varying from 200-220g with experimental brain injuries ("weight-drop method"). The concentration of NSE and GFAP and the level of cerebral edema were estimated after the injury in animals that were treated with pyrimidine derivative $(50 \mathrm{mg} / \mathrm{kg})$ and choline alfoscerate $(50 \mathrm{mg} / \mathrm{kg})$.

Results: The level of biomarkers in group of negative control was elevated. After the treatment the experimental animals with pyrimidine derivative GLY and choline alfoscerate the concentration of NSE and GFAP decreased as well as cerebral edema.

Conclusion: Administration of GLY one hour after injury at a dose 50 $\mathrm{mg} / \mathrm{kg}$ minimizes cerebral edema and the damage of neurons.

Copy Right, IJAR, 2019,. All rights reserved.

\section{Introduction:-}

Traumatic Brain Injury (TBI) is a critical public health and a major socio-economic problem throughout the world. So far, it is the leading cause of morbidity and mortality among the young people. $(2,10,13)$.

Worldwide, TBI affects 10 million individuals annually. Approximately, 1.7 million people in United States sustain a TBI each year, resulting in more than 235,000 hospitalizations and 50,000 deaths (8).

TBI initiates a complex of series of neurochemical signaling events that includes: excitotoxicity, hypoxemia, oxidative stress, ischemia, inflammation and apoptosis (8).

One of the leading pathophysiological pathways that increase intracranial pressure and determine the progression of trauma is cerebral edema. It's a predictive factor of poor outcome among survivors and risk of death following TBI (4). 
Although the survival rate of TBI has been improving continuously for decades, particularly in developing countries, there has been no real progress in the prevention and management of the lifelong impairments induced by its trauma. Consequently, a better understanding of the mechanism of the TBI would help in the selection of most appropriate treatment protocol, thus it becomes more cost effective and lifesaving for several cases $(3,10,12)$.

Early detection and treatment of TBI may improve the outcome and may reduce long-term cognitive complications. Tissue damage can be monitored by several ways that include, clinical assessment scales, neurological examination, or computed tomography.

Last decades, estimation of serum markers can be helpful in providing severity of TBI, its prognosis, and efficacy of therapy. The most promising biomarkers of brain damage are Glial Fibrillary Acid Protein (GFAP) and NeuronSpecific Enolase (NSE). NSE is a marker of axonal injury that released by cell destruction, and predictable in neurological outcomes. GFAP is a specific biomarker for the brain damage and useful in predicts the morbidity and mortality $(2,5,7,9)$.

The aim of this study was to estimate the efficiency of pyrimidine derivative GLY and choline alfoscerate on the development of the brain edema and to monitor the response of the therapy, by the estimation of GFAP and NSE concentrations.

\section{Materials and methods:-}

For our experiments, we used the weight drop method, due to its feasibility, simplicity, as well as the ability to control the level of injury (by adjusting the height and the mass of the weight dropped). The impact weight $150 \mathrm{~g}$, it was positioned at the top of the aluminum tube, height $50 \mathrm{~cm}$ on the head. In all studies, male Wistar rats were used. The weight ranged between 200-220g (1). All work was performed in accordance with the ethical and national guidelines for animal experiments. All animals were anaesthetized by using intra-abdominal injection of chloral hydrate $(350 \mathrm{mg} / \mathrm{kg})$. The animals were randomly assigned to four groups:

1. The sham-operated group (positive control) with no treatment $(n=20)$

2. The TBI group (negative control) without treatment $(n=20)$

3. Group that was treated with pyrimidine derivative $(n=20)$

4. Group with choline alfoscerate treatment $(n=20)$

After 0.5 hours after anaesthesia, experimental groups of animals were exposed to the weight dropped method. Approximately 1 hour after injury, all experimental animals, except the negative control group and sham-operated group, were injected with derivative of pyrimidine GLY $(50 \mathrm{mg} / \mathrm{kg})$ and choline alfoscerate $(50 \mathrm{mg} / \mathrm{kg}) \mathrm{during}$ the following 3 days after the trauma. On the fourth day after injury, blood samples were collected and stored at room temperature for 15 minutes. Then samples were centrifuged and were preserved at the temperature $-80{ }^{0} \mathrm{C}$ for detection. The serum levels of NSE and GFAP were detected by Tecan "Infinite F50" according manufacturer's instructions ("Cloud clone").

The brain water content (BWC) was calculated by wet-dry method. (4)

Analyses were performed using Statistika 6.0. One-way analyses of variance [ANOVA] were used to compare the differences among the control, the TBI group without treatment, and groups with pharmacological support. P<0.05 was considered to indicate a statistically significant difference.

\section{Results:-}

After exposure to the trauma, no rodent died in the groups. After TBI, serum levels of GFAP and NSE were elevated in a group of negative control and were significantly higher than in the sham-operated group $(\mathrm{P}<0.05)$. In the group of animals, with choline alfoscerate correction, NSE concentration decreased $113,9 \%(\mathrm{P}<0.05)$ compared to group 2. In group 3 that was treated with GLY serum, NSE value was $7.36 \pm 0.46 \mathrm{ng} / \mathrm{ml}$ and less then group of negative control $(\mathrm{P}<0.05)$.

The level of GFAP concentration in group of negative control reached $29,8 \pm 0,92 \mathrm{ng} / \mathrm{ml}$ and it was significantly higher than sham-operate group $(\mathrm{P}<0.05)$. The correction of TBI in group 3 and 4 decreased the level of biomarker on 1.96 and on 1.65 times $(\mathrm{P}<0,05)$. 


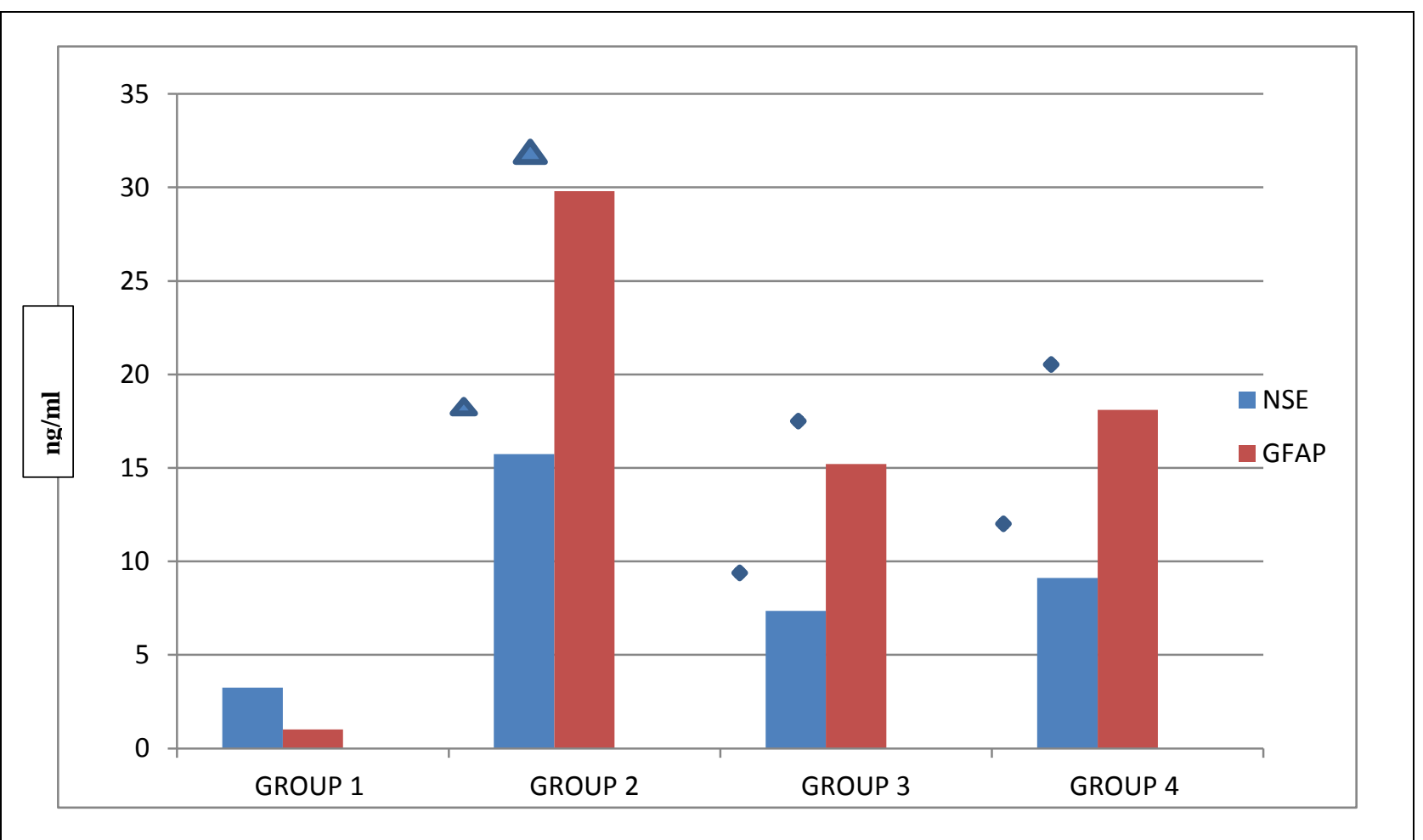

FIGURE 1:- Course of the changes of NSE and GFAP levels after TBI

1. Significance of differences in comparison with group without treatment $(\mathrm{P}<0.05)$

2. Significance of differences in comparison with sham-operated animals $(\mathrm{P}<0.05)$

Regarding Brain Edema, BWC in the group of negative control was $80,03 \pm 2,1 \%$ and in the group of sham-operated animals was $75,23 \pm 1,8 \%(\mathrm{P}, 0.05)$. After correction by pyrimidine derivative, BWC decreased and was estimated at $77,33 \pm 1,5 \%$, as well as in group $476,37 \pm 1,8 \%$.

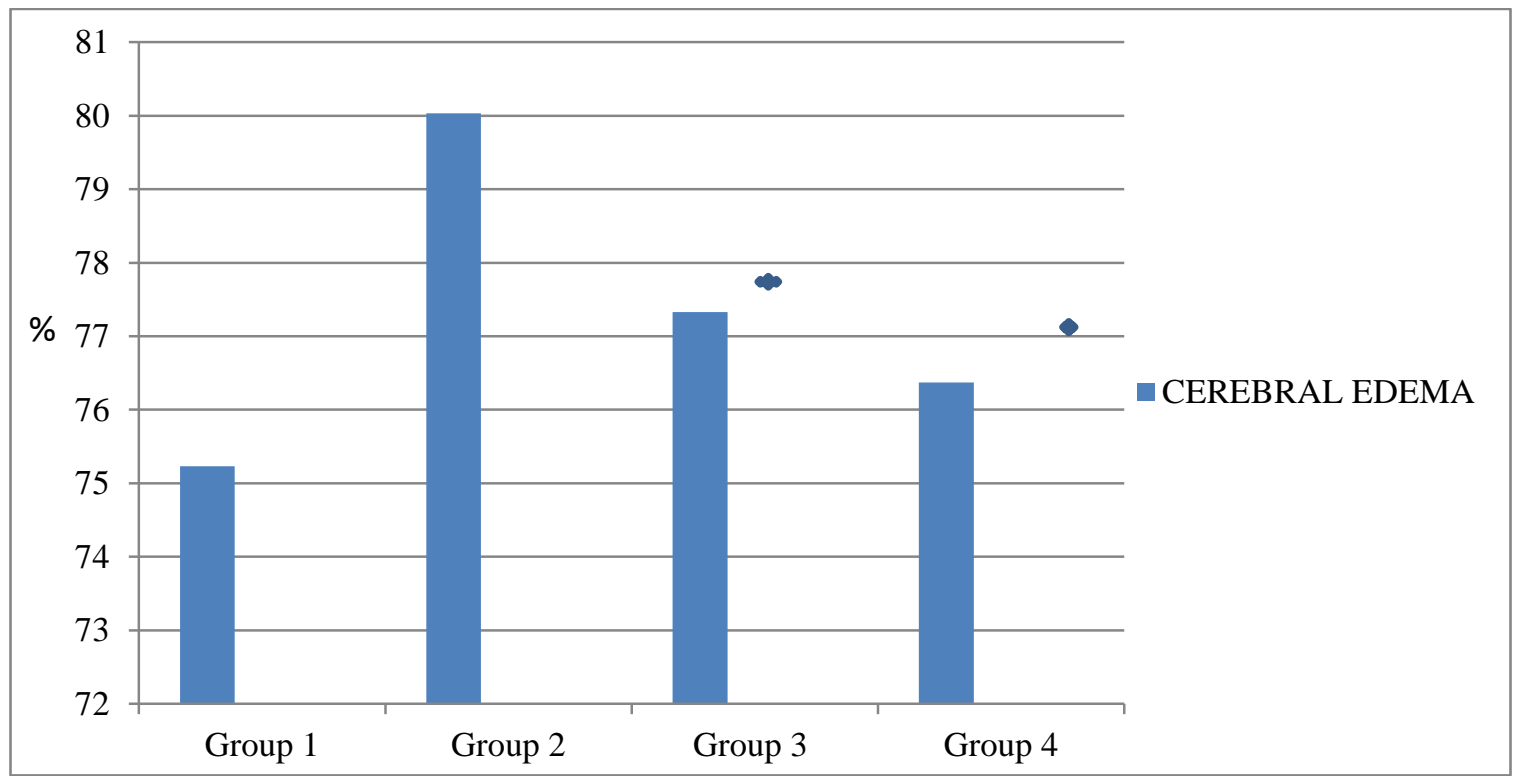

Figure 2:-Cerebral Edema in animals with experimental TBI.

Significance of differences in comparison with group without treatment $(\mathrm{P}<0.05)$. 


\section{Discussion:-}

Brain injury initiates a complex of neurochemical signaling events that are classified as a primary (occurring at the time of mechanical impact), and a secondary (occurring minutes to days after the injury), its interaction may give a rise to microhemorrhages, hematomas, swelling, apoptosis, oxidative stress, inflammation, and vascular necrosis. Several biomarkers have been identified to reflect such biological response; such as, neuron specific enolase (NSE), glial fibrillary acid protein (GFAP). Although these proteins are still being assessed, their combination can provide valuable information for estimating the severity of the trauma, hence the given guideline for proper treatment (4).

GFAP, is the major component of the cytoskeleton of astrocytes. After trauma is released from the degenerating brain into surrounding tissues and can provide the diagnostic information in predicting outcome after the brain injury (5).

NSE is mainly localized in the neurons. Some authors show that elevation of this biomarker for the first 3 days after the trauma and slowly decrease over the next two weeks $(6,11)$.

Pyrimidine derivatives, was shown to have an antioxidant, antihypoxic, and anti-apoptotic activity (1). In this experimental study, we found a significant decrease of cerebral edema when compound GLY was administered, at the same time the concentration of NSE and GFAP was significantly less than in the group without treatment. In experimental model, we found a significant decrease in brain edema, in groups with GLY and choline alfoscerate administration.

We hypothesis that derivatives of pyrimidine decreases the permeability of Blood Brain Barrier and improves the neurons function. This will minimize the cognitive impairment and enhances the outcome.

\section{Conclusion:-}

It is concluded clearly that the compound GLY is advisable to further study in the treatment experimental brain injury.

\section{Acknowledgements and Funding:-}

This research received no specific grant from any funding agency in the public, commercial, or not-for-profit sectors.

\section{Conflicts of Interest}

The authors statement no conflict of interest with submitted manuscript.

\section{Abbreviations}

NSE- neuron-specific enolase, GFAP- glial fibrillary acid protein, TBI- traumatic brain injury

\section{Summary:}

1. In animals with experimental TBI, we observed an elevated serum level of biomarkers of neuronal damage and cerebral edema.

2. The treatment with compound GLY leads to a decrease of GFAP/ NSE of 113, 9\%/ 96,1\% compare to negative control group.

3. Our study suggests that treatment the experimental animals with pyrimidine derivative GLY has a positive neuroprotective effect and it decreases the cerebral edema. 


\section{References:-}

1. Voronkov A.V., Kalashnikova S.A., Khoury E.I. The traumatic brain injury modeling by "the weight-dropped method"// Современные проблемы науки и образования. - 2016. - № 5.;URL: https://www.scienceeducation.ru/ru/article/view?id=25242.

2. 2. Dambinova Svetlana A., Ronald L. Hayes Biomarkers for Traumatic Brain Injury.2012: 3-11.

3. Feala Jacob D., Mohamed Diwan M. AbdulHameed Systems Biology approaches for discovering biomarkers for traumatic brain injury, Journal of Neurotrauma. 2013; 30:1101-1116.

4. Ismet Topcu The effects of lornoxicam on brain edema and blood brain barrier following diffuse traumatic brain injury in rats Turkish Journal of Trauma and Emergency Surgery. 2013; 19(4): 294-298.

5. Jun Ding, Zhengang Ding Proteomic Analysis after Traumatic Brain Injury in Rats: The Research for Potential Biomarkers//Arq Neuropsiquiatr. 2015;73(4):342-349.

6. L.Fletcher, C.C.Rider, and C.B. Taylor Enolase isoenzymes. Chromatographic and immunological characteristics of rat brain enolase. Biochim Biophys Acta.1976; 452:245-252.

7. Pelinka, Linda E.; Hertz Nonspecific increase of systemic NSE after trauma;clinical and experimental findings Basic Science aspects. 2005; 24(2):119-123.

8. Risdall Jane E., David Menon Traumatic Brain Injury / Philosophical Transactions of Royal Society. 2011; 366: 241-250.

9. Tomaszewski Dariusz Biomarkers of Brain Damage and Postoperative Cognitive. Hindawi Publishing Corporation BioMed Research International.2015. doi:10.1155/2015/402959.

10. X. Anton Alvarez, Jesus Figueroa Peptidergic Drugs for the Treatment of Traumatic Brain Injury// Future Neurology.2013; 8(2):175-192.

11. U.E. Pleins, M.C. Morganty-Kossmann S-100ß reflects the extend of injury and outcome, whereas neuronal specific enolase is a better indicator neirounflammation in patients with severe traumatic brain injury. J. Neurotrauma.2001; 18:491-408.

12. Sean Shen, Rachel R. Ogorzalek Loo Addressing the Needs of Traumatic Brain Injury with Clinical Proteomics.2014;11:11.

13. Ovsyannikov D.M. Social and Epidemiological Aspects of Craniocerebral Trauma. 2012;8(3). 